A $C \underset{\text { publications }}{\mathrm{C}}$ Rec. Nat. Prod. 15:1 (2021) 25-34

records of natural

products

\title{
Salvianolic Acid B Attenuated Ischemia/Reperfusion- Induced Brain Injury in Mice by Inhibiting Reactive Oxygen Species-Mediated Inflammation
}

\author{
Chiyeon Lim ${ }^{1}$, Chang-Hyun Kim $\oplus^{1}$, Se-Hyun $\operatorname{Lim} \oplus^{2 *}$ and Suin $\mathrm{Cho} \oplus^{3^{*}}$ \\ ${ }^{I}$ College of Medicine, Dongguk University, Goyang, 10326, Republic of Korea \\ ${ }^{2}$ School of Public Health, Far East University, Eumsung, 27601, Republic of Korea \\ ${ }^{3}$ School of Korean Medicine, Pusan National University, Yangsan, 50612, Republic of Korea
}

(Received March 06, 2020; Revised May 19, 2020; Accepted May 23, 2020)

\begin{abstract}
Salvianolic acid B (SAB) is one of main components of the dried roots and rhizomes of Salvia miltiorrhiza Bunge (RSM), which is widely used as a medicinal herb in East-Asia. According to traditional Korean medicine, RSM promotes blood flow, and thus, we investigated the effects of administering SAB after ischemia/reperfusion (I/R)-induced brain injury in a mouse model. I/R-induced brain injury was induced by middle cerebral artery occlusion (MCAO) for $2 \mathrm{~h}$. SAB was orally administered to mice twice after 3 and $6 \mathrm{~h}$ of occlusion at 5,15 , or $45 \mathrm{mg} / \mathrm{kg}$ body weight. Administration of $\mathrm{SAB}$ at $45 \mathrm{mg} / \mathrm{kg}$ significantly reduced infarct volumes and edema indices and suppressed interleukin 1 beta (IL-1 $\beta$ ), tumor necrosis factor alpha (TNF- $\alpha$ ), and reactive oxygen species (ROS) levels in brain ipsilateral hemispheres. SAB also down-regulated Mn-SOD protein expression in affected brain tissues after I/R-induced brain injury. Our results indicate the neuro-protective effects of salvianolic acid B are due to its anti-inflammatory effect mediated by intra-cellular superoxide scavenging.
\end{abstract}

Keywords: Salvianolic acid B; Salvia miltiorrhiza; ischemia/reperfusion injury; inflammation; brain edema. (C) 2020 ACG Publications. All rights reserved.

\section{Introduction}

About $60 \%$ of deaths from cardiovascular disease, half of all coronary artery deaths, and twothirds of stroke deaths occur in low and middle-income countries. Because $87 \%$ of all stroke cases are ischemic, stroke can cause many morbid conditions and disorders that place significant strains on healthcare systems [1,2]. To date, recombinant tissue plasminogen activator (r-tPA) is the only standard treatment approved by the US FDA for acute ischemic stroke, but its clinical application is limited to within 4-5 $\mathrm{h}$ after stroke [3-6].

Brain damage from ischemia/reinfusion ( $\mathrm{I} / \mathrm{R})$ is the result of complex pathophysiological processes, but oxidative stress and inflammation are thought to be two major factors responsible for cell death from I/R. Excessive accumulations of reactive oxygen species (ROS) have been shown to induce

Corresponding authors: E-Mail: sicho@pusan.ac.kr (S.Cho); Phone:082-51-510-8457 Fax:082-51-510-8420; favor119@hanmail.net (S.H. Lim). 
cell death by modulating a series of intracellular signaling pathways $[7,8]$. Furthermore, inflammatory biomarkers such as cytokines, chemokines, and acute phase reactants control inflammatory reactions, and increases in inflammatory mediators, such as interleukin 1 beta (IL-1 $\beta$ ) and tumor necrosis factor alpha (TNF- $\alpha$ ), are known to be associated with an increased risk of stroke [7,9].

In the past few decades, hundreds of drugs that target the many pathophysiological mechanisms of stroke have failed clinical trials. However, studies performed to date provide important information on the role of immunity in all stages of stroke $[9,10]$, and as a result, various immuno-targeting therapies are being developed and tested.

Salvia miltiorrhiza Bunge (RSM) root is a well-known, widely used herbal medicine in EastAsia for the treatment of cardiovascular diseases, and salvianolic acid B (SAB) is a major functional phenolic component in RSM [11-13]. Recently, Yang et al. reported SAB protected cardiomyocytes from oxygen and glucose deprivation (OGD) [14], and Fan et al. reported SAB inhibited ROS and inflammatory factor production by suppressing the activations of microglia and astrocytes in 1-h mouse middle cerebral artery occlusion (MCAO) model of I/R-induced brain injury [15].

Recent preliminary studies conducted in our laboratory showed that SAB reduced total infarction volumes in ipsi-lateral hemispheres, but our results differed from those of Fan et al. [15], and thus, we conducted this study by changing experimental conditions. In addition, we modified the experimental mouse model and refined experimental protocols to improve reproducibility. In the present study, we explored the anti-inflammatory effects of SAB in a 2-h mouse middle cerebral artery occlusion (MCAO) model of I/R-induced brain injury.

\section{Materials and Methods}

\subsection{Reagents}

Phosphate buffered saline (PBS) was purchased from Bio Basic Inc. (Markham, Ontario, Canada). 2,3,5-triphenyl-tetrazolium chloride (TTC) and protein extraction solution were purchased from iNtRON (Seongnam, Gyeonggi, Korea). Primary antibodies for manganese superoxide dismutase (Mn-SOD) and $\beta$-actin were from Cell Signaling Technology (Danvers, MA, USA). Goat polyclonal secondary anti-mouse IgG antibody was obtained from Enzo Life Sciences Inc. (Farmingdale, NY, USA). Bovine serum albumin (BSA) standard and enhanced chemiluminescence (ECL) western blotting chemiluminescent substrate were purchased from Thermo Fisher Scientific (Waltham, MA, USA).

\subsection{Ischemic Stroke Mouse Model}

Male adult specific-pathogen-free (SPF) C57BL/6 mice weighing 22-25 g were obtained from Daehan Biolink Co. (Chungbuk, Korea). Animals were housed in a temperature/humidity-controlled animal facility under a $12 \mathrm{~h}$ light/dark cycle and provided food and water ad libitum. All animal experiments were conducted in accordance with the recommendations of the Declarations of Helsinki and Tokyo, and the experiments were approved and regulated by the Ethics Committee of Pusan National University. (Approval Numbers, PNU-2017-1752 and PNU-2018-2113).

Middle cerebral artery occlusion (MCAO) induced ischemic stroke was performed as previously described [16]. Briefly, mice were anesthetized with $2 \%$ isoflurane and maintained with $1.5 \%$ isoflurane in a mixture of $70 \% \mathrm{~N}_{2} \mathrm{O}$ and $30 \% \mathrm{O}_{2}$. The left common carotid artery (LCCA) was exposed and an 11mm-long 8-0 monofilament nylon suture (Ethicon Inc. NJ, USA) was inserted and advanced into the internal carotid artery (ICA) to occlude the MCA. Two h later the suture was removed to allow initiate blood flow reperfusion (Figure 1). After the operation, animals were placed in recovery chamber and maintained at $37^{\circ} \mathrm{C}$ for $30 \mathrm{~min}$. 


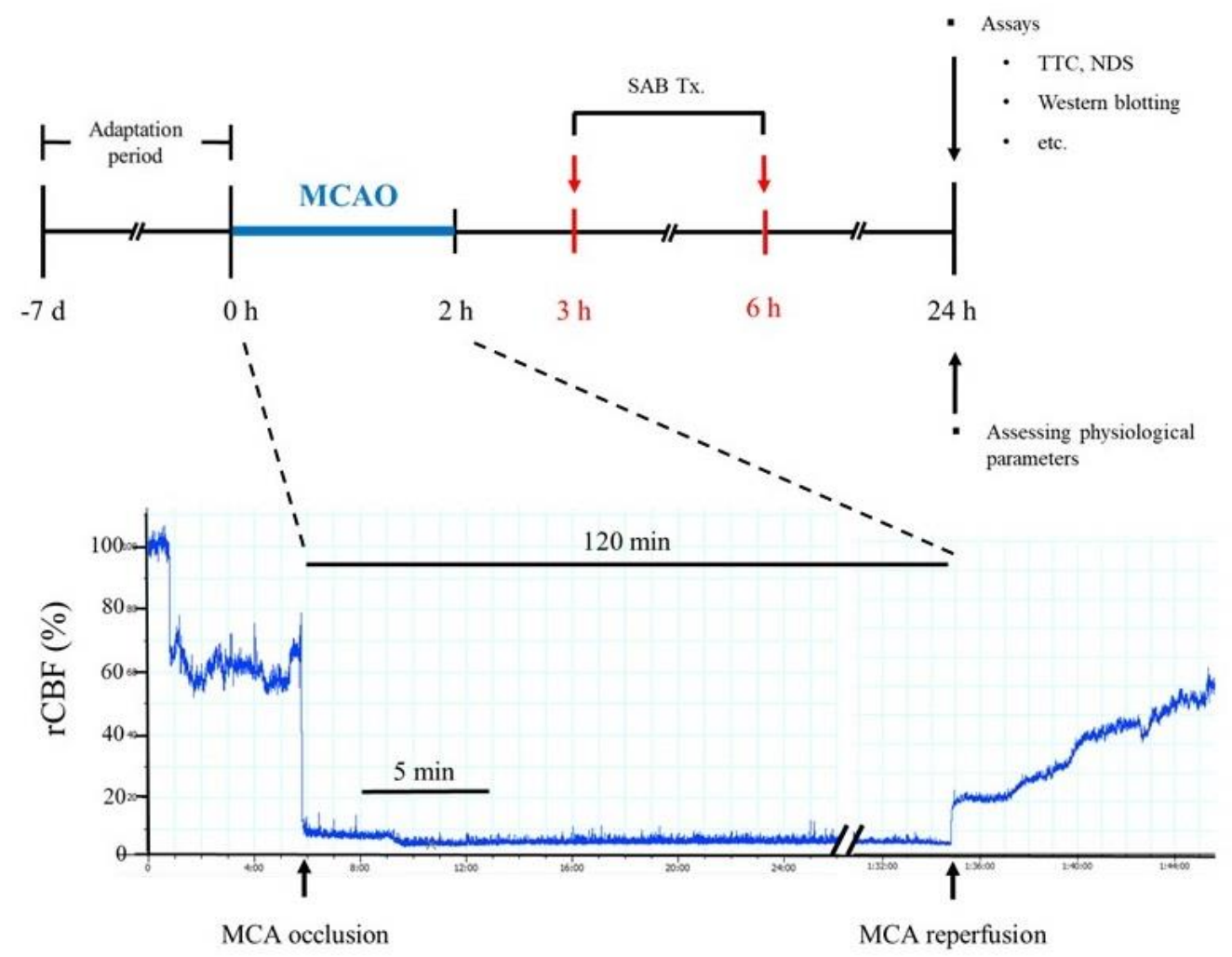

Figure 1. Design of the experimental I/R-induced brain injury model. Brain injury was induced in mice by MCAO. Mice were treated with SAB twice 3 and $6 \mathrm{~h}$ after MCAO commencement. MCAO was maintained for $120 \mathrm{~min}$, and relative cerebral blood flow was monitored by laser-Doppler flowmetry.

\subsection{SAB Treatment}

SAB (purity > 94\%) was purchased from Merck Co. (Kenilworth, NJ, USA); high-performance liquid chromatography (HPLC) fingerprinting data was used to confirm its purity (see supporting information, Figure S1).

$\mathrm{SAB}$ was prepared for oral administration to mice by dissolving it in distilled water. This solution was diluted with $0.9 \%$ normal saline, passed through a $0.45-\mu \mathrm{m}$ pore size syringe filter for sterilization, and adjusted to concentrations of 5,15 , and $45 \mathrm{mg} / \mathrm{kg}$, respectively.

Mice were divided into 5 groups of 5 mice, that is, the sham-operated group (the sham group), the MCAO-operated group (the MCAO group), and three MCAO-operated SAB groups treated with 5, 15 , or $45 \mathrm{mg} / \mathrm{kg}$ (the SAB 5, 15, and 45, respectively). Mice in the SAB-treated groups received SAB orally at the designated concentrations at 3 and $6 \mathrm{~h}$ after commencing MCAO (Figure 1). Mice in the sham and MCAO groups were administered the same volume of normal saline.

\subsection{Physiological Parameter Measurements}

Mice were weighed daily during the experimental period and blood was collected from abdominal aortas under deep anesthesia $24 \mathrm{~h}$ post-MCAO. To measure serum electrolytes, blood samples were centrifuged at $1,500 \times g$ for $15 \mathrm{~min}$ at $4^{\circ} \mathrm{C}$. An electrolyte analyzer (Dri-Chem 3500i, Fuji, Japan) was used to measure serum levels of sodium $\left(\mathrm{Na}^{+}\right)$, potassium $\left(\mathrm{K}^{+}\right)$, and chloride $\left(\mathrm{Cl}^{-}\right)$to detect and eliminate animals with a possible electrolyte imbalance, which might have affected brain edema index and edema-induced infarct sizes. 


\subsection{Infarct Volume Measurement}

After $2 \mathrm{~h}$ of ischemia and $22 \mathrm{~h}$ of reperfusion, brains were removed, sliced into 10 coronary slices $\left(1 \mathrm{~mm}\right.$ thick), stained at room temperature $\left(25^{\circ} \mathrm{C}\right)$ with $2 \%$ TTC for $17 \mathrm{~min}$, and immersed in $10 \%$ neutral buffered formalin (NBF) for $2 \mathrm{~h}$. Infarction areas in these sections used to calculate percent infarct volumes, as described previously [16], using a digital camera (Canon 20D, Canon Korea, Korea) and ImageJ software (NIH, Maryland, USA).

\subsection{Evaluation of Neurological Deficit Scores (NDSs)}

Twenty-four $\mathrm{h}$ after MCAO, the following five-point scale was used to determine neurological deficit scores: 0 , no neurological deficit; 1 , incomplete extension of right forefoot and lowering of grip when the tail was pulled; 2 , voluntary movement in all directions and turning right when the tail was pulled; 3 , walking or turning to the right and appearing sensitive when the tail was stimulated; 4 , no reaction to stimulation or stroke-related death.

\subsection{Brain Edema Area Calculation}

Brain edema areas were calculated by dividing total infarction volumes in ipsilateral hemispheres by brain edema indices using TTC-stained brain section. The formula used to calculate brain edema indices was as follows; hemisphere

Brain edema index $=$ total volume of ipsilateral hemisphere $/$ total volume of contralateral

\subsection{Inflammatory Cytokine Analysis}

Brains were carefully separated from skulls, dissected, and ischemic ipsilateral hemispheres were homogenized in ice cold PBS ( $\mathrm{pH} 7.4,5 \% \mathrm{w} / \mathrm{v})$. Obtained homogenates were clarified at 10,000 $\times \mathrm{g}$ at $4^{\circ} \mathrm{C}$ for $10 \mathrm{~min}$, and supernatants were subjected to enzyme-linked immunosorbent assay (ELISA). Levels of IL-1 $\beta$ and TNF- $\alpha$ in ipsilateral hemispheric brain tissues were measured by ELISA using a commercially available kit (Abcam, Cambridge, MA, USA). Absorbances of reaction products were measured at $450 \mathrm{~nm}$ using a microplate reader.

\subsection{Determination of ROS Production}

Dichlorofluorescein diacetate (DCFH-DA) was used to determine ROS levels in tissue homogenates, as previously described [17]. Tissue homogenates were incubated at $37^{\circ} \mathrm{C}$ for $30 \mathrm{~min}$ with $1 \mathrm{mM}$ DCFH-DA, and a fluorescent microplate reader was used to measure absorbances using an excitation wavelength of $485 \mathrm{~nm}$ and an emission wavelength of $535 \mathrm{~nm}$.

\subsection{Western Blot Analysis}

Expression levels of Mn-SOD in ipsilateral hemispheres were evaluated by western blotting as we previously described, with some modification [18]. In brief, mice were euthanized $24 \mathrm{~h}$ after MCAO by $\mathrm{CO}_{2}$ inhalation and brains were carefully isolated and placed on ice-cold glass. Ischemic ipsilateral hemispheres were dissected, homogenized, and incubated in PBS containing $150 \mathrm{mM} \mathrm{NaCl}, 1 \mathrm{mM}$ EDTA, $50 \mathrm{mM}$ Tris and 1:100 (v/v) of proteinase inhibitor. After centrifugation $(13,250 \times \mathrm{g})$ at $4{ }^{\circ} \mathrm{C}$ for $10 \mathrm{~min}$, residues were removed and total proteins were obtained from supernatants using a protein extraction solution. Total protein contents were then determined using a protein assay kit (Quick Start, Bio-Rad, USA). In addition, equal amounts of total proteins were separated in 10\% sodium dodecyl sulfate-polyacrylamide gels (SDS-PAGE) and transferred to polyvinylidene difluoride (PVDF) membranes (Millipore, Darmstadt, Germany), which were then blocked with 5\% skim milk in Trisbuffered saline containing $0.1 \%$ Tween 20 (TBST, Sigma-Aldrich, USA) for $1 \mathrm{~h}$ at $25^{\circ} \mathrm{C}$, incubated overnight with primary antibodies (anti-AQP-4, 1:500; anti- $\beta$-actin, 1:1000) (Cell Signaling, MA, USA) at $4^{\circ} \mathrm{C}$, washed with TBST, and incubated with horseradish peroxidase (HRP)-conjugated secondary 
antibody for $2 \mathrm{~h}$ at $25^{\circ} \mathrm{C}$. After washing three times with TBST, blots were developed using an ECL kit and detected using a photosensitive luminescent analyzer system (Amersham Imager 600, GE Healthcare, IL, USA). ImageJ software was used to analyze blot signal strengths to determine the relative expression of Mn-SOD versus $\beta$-actin.

\subsection{Statistical Analysis}

One-way analysis of variance (ANOVA) followed by Holm-Sidak post-hoc analysis was used to determine the significances of intergroup differences. Holm-Sidak analysis was used to evaluate differences between groups for pairwise multiple comparisons when data were non-normally distributed. The analysis was performed using Sigmaplot v12.0 software (Systat Software Inc., CA, USA) and results are presented as means \pm standard deviations (SDs). Statistical significance was accepted for $\mathrm{p}$ values $<0.05$.

\section{Results and Discussion}

\subsection{Effects of SAB on Infarct Percentage and Neurological Deficit Scores}

Representative images of TTC-stained brain sections and percentage infarct volumes are shown in Figure 2A. MCAO for $2 \mathrm{~h}$ caused ischemia-induced damage in ipsilateral brain hemispheres (mean infarct percentage, $54.87 \pm 8.50 \%$ ), whereas sham surgery did not. Treatment with $45 \mathrm{mg} / \mathrm{kg} \mathrm{SAB}$ at 3 and $6 \mathrm{~h}$ after MCAO commencement significantly reduced infarct volumes (by $35.20 \pm 7.16 \%$ ) versus the sham group. MCAO significantly increased NDSs and treatment with SAB resulted in no improvement (Figure 2B).
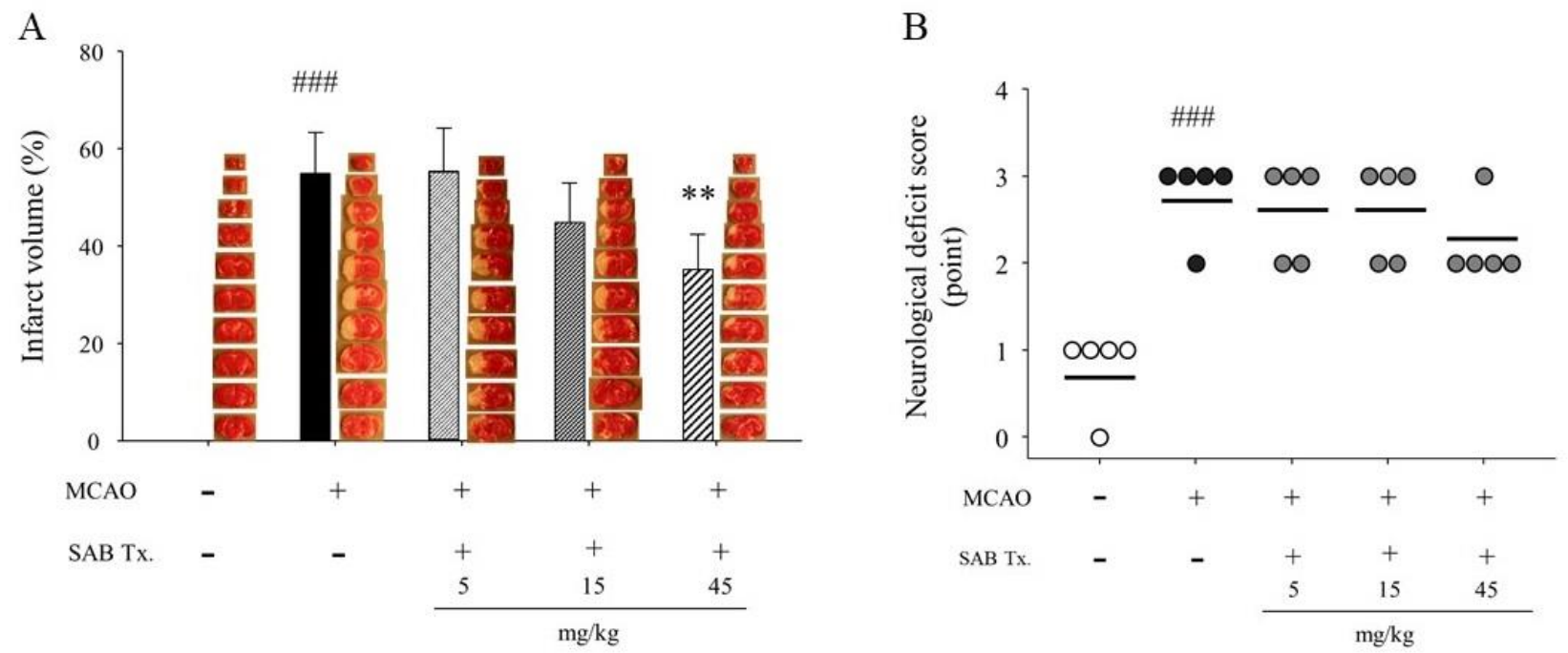

Figure 2. Representative images and total infarction volumes of brain sections (A), and neurological deficit scores (NDSs) (B) of mice in the five study groups.

Treatment with SAB ( $45 \mathrm{mg} / \mathrm{kg}$ body weight) at 3 and $6 \mathrm{~h}$ after MCAO significantly decreased infarct volumes (horizontal bar, mean value), but did not improve NDSs. Results are presented as means \pm SDs. \#\#\#p<0.001 vs sham controls. ${ }^{* *} \mathrm{p}<0.01$ vs the MCAO group; $\mathrm{n}=5$ per group.

We previously described an improved ischemic stroke mouse model [16], in which occlusion times are correlated with cerebral infarction areas. When we reviewed the study by Fan et al. [15], the infarction area they observed correlated with 90 or $120 \mathrm{~min}$ of occlusion in our model, though they only used 60 min of occlusion. Fan et al. performed MCAO using the same strain as used in the present study, but used a 7-0 suture to block blood flow by intra-luminal insertion, whereas we used an 8-0 suture. We believe that the thicker suture used created a larger brain infarction volume despite the shorter ischemic 
time. Furthermore, Fan et al. administered SAB (10, 20, 40, or $60 \mathrm{mg} / \mathrm{kg}$, respectively) once immediately after establishing reperfusion, and we administered $(5,15$, or $45 \mathrm{mg} / \mathrm{kg}$ ) twice. Despite these differences, SAB reduced the amount of infarction in both studies, but Fan et al. reported that NDSs were improved by SAB administration, whereas we did not do so. Accordingly, we checked the basic action mechanism of SAB using different administration conditions. The results obtained confirmed our earlier findings that when $\mathrm{SAB}$ was administered twice after cerebral I/R injury, it reduced the amount of infarction (Figure 2A), but did not reduce NDSs (Figure 2B).

\subsection{Effects of SAB on Physiological Parameters and Brain Edema}

After I/R body weights tended to decrease in MCAO operated groups, but not significantly (data not shown). Serum electrolyte levels in the five study groups were not significantly different postMCAO (Figure 3A), indicating no physiological changes such as edema and brain damage occurred that might have affected intergroup comparisons.

Results on brain edema indices (Figure 3B) correlated with overall infarction volumes, suggesting the severity of damage post-MCAO was correlated with the extent of cerebral edema, which in turn, suggests brain edema be viewed as an important treatment target in cases of brain injury, and that SAB might be considered a potential treatment for cerebral edema.
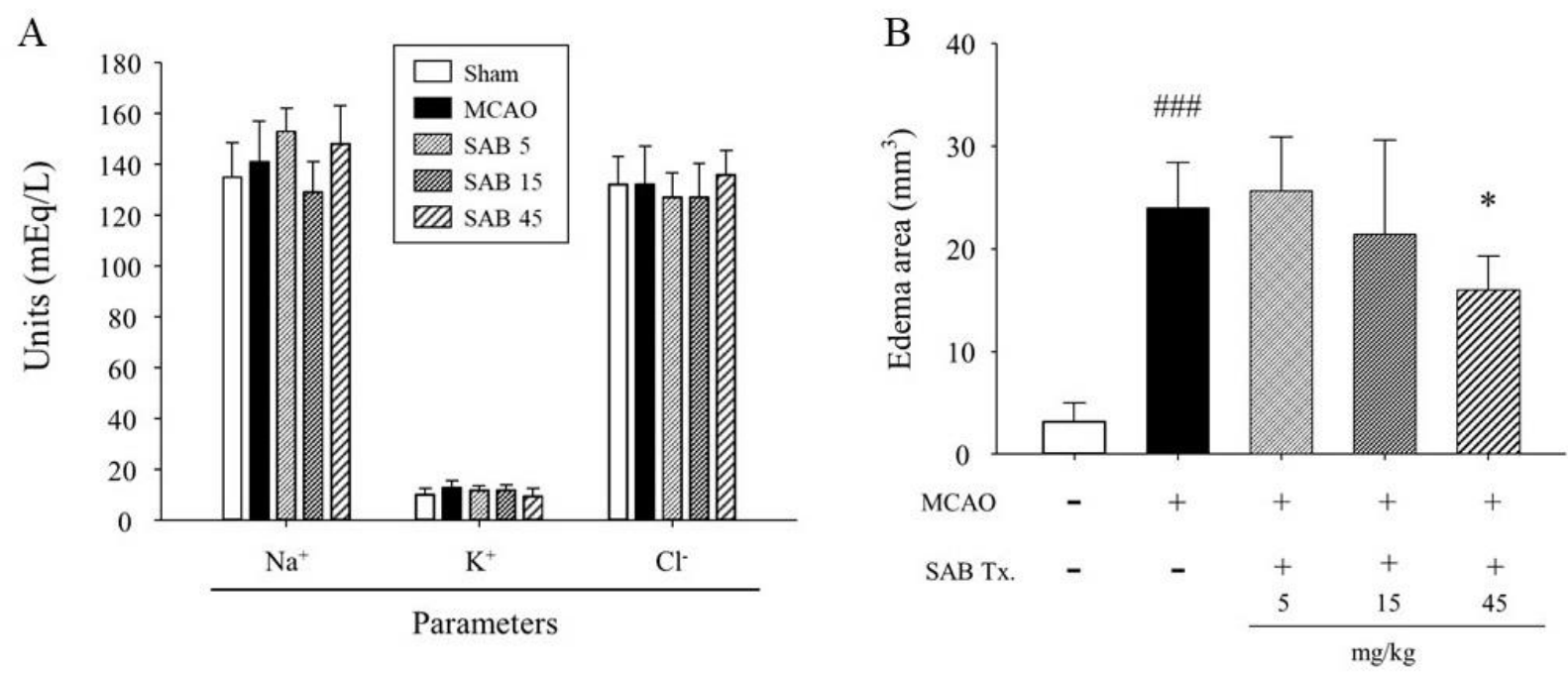

Figure 3. Influence of MCAO on physiological parameters (A) and effect of SAB on edema in ischemic ipsilateral hemispheres $(\mathbf{B})$.

Serum samples were obtained and concentrations of electrolytes such as sodium $\left(\mathrm{Na}^{+}\right)$, potassium $\left(\mathrm{K}^{+}\right)$, and chloride $\left(\mathrm{Cl}^{-}\right)$were measured. Treatment with $\mathrm{SAB}$ twice at $45 \mathrm{mg} / \mathrm{kg} 3$ and $6 \mathrm{~h}$ after MCAO commencement significantly suppressed MCAO-induced increases in brain edema (B). Results are presented as means \pm SDs. $\# \#$ \# $<0.001$ vs the sham group, ${ }^{*} \mathrm{p}<0.05$ vs the MCAO group; $\mathrm{n}=5$ per group.

\subsection{Effects of SAB on Pro-inflammatory Cytokine Levels and ROS Production in Brain Tissues}

The ischemic ipsilateral hemispheres of mice in the MCAO group had higher concentrations of IL-1 $\beta(489.40 \pm 78.69 \mathrm{pg} / \mathrm{mL})$ and TNF- $\alpha(389.00 \pm 67.10 \mathrm{pg} / \mathrm{mL})$ than those of mice in the sham group (IL1 $\beta, 191.21 \pm 37.41 \mathrm{pg} / \mathrm{mL}$; TNF- $\alpha, 144.23 \pm 34.66 \mathrm{pg} / \mathrm{mL}$ ). However, treatment with $45 \mathrm{mg} / \mathrm{kg}$ SAB twice significantly suppressed the expressions of IL-1 $\beta(380.80 \pm 56.14 \mathrm{pg} / \mathrm{mL})$ and TNF- $\alpha(258.23 \pm$ $48.87 \mathrm{pg} / \mathrm{mL}$ ) (Figure 4A, B).

To examine antioxidative effect of SAB, ROS levels in ischemic ipsilateral hemispheres were measured. ROS levels were significantly higher in the MCAO group $(197.25 \pm 9.78 \%)$ than in the sham group $(104.58 \pm 16.13 \%)$, but were significantly lower in the SAB 15 and SAB 45 groups than in the MCAO group (Figure 4C).

Since oxidative stress and pro-inflammatory cytokines elicited by inflammation mediate tissue damage, ROS play important roles in the progression of inflammatory disorders [19]. The pathogenic 
roles of IL-1 $\beta$ and TNF- $\alpha$ in rodent models of stroke are well established. For example, exogenous administration of IL- $1 \beta$ increased brain edema in mice, whereas IL- $1 \beta$ knockout reduced infarction sizes [20]. In another study, circulating levels of TNF- $\alpha$ were found to be correlated with infarct size and severity of neurological impairment [21].
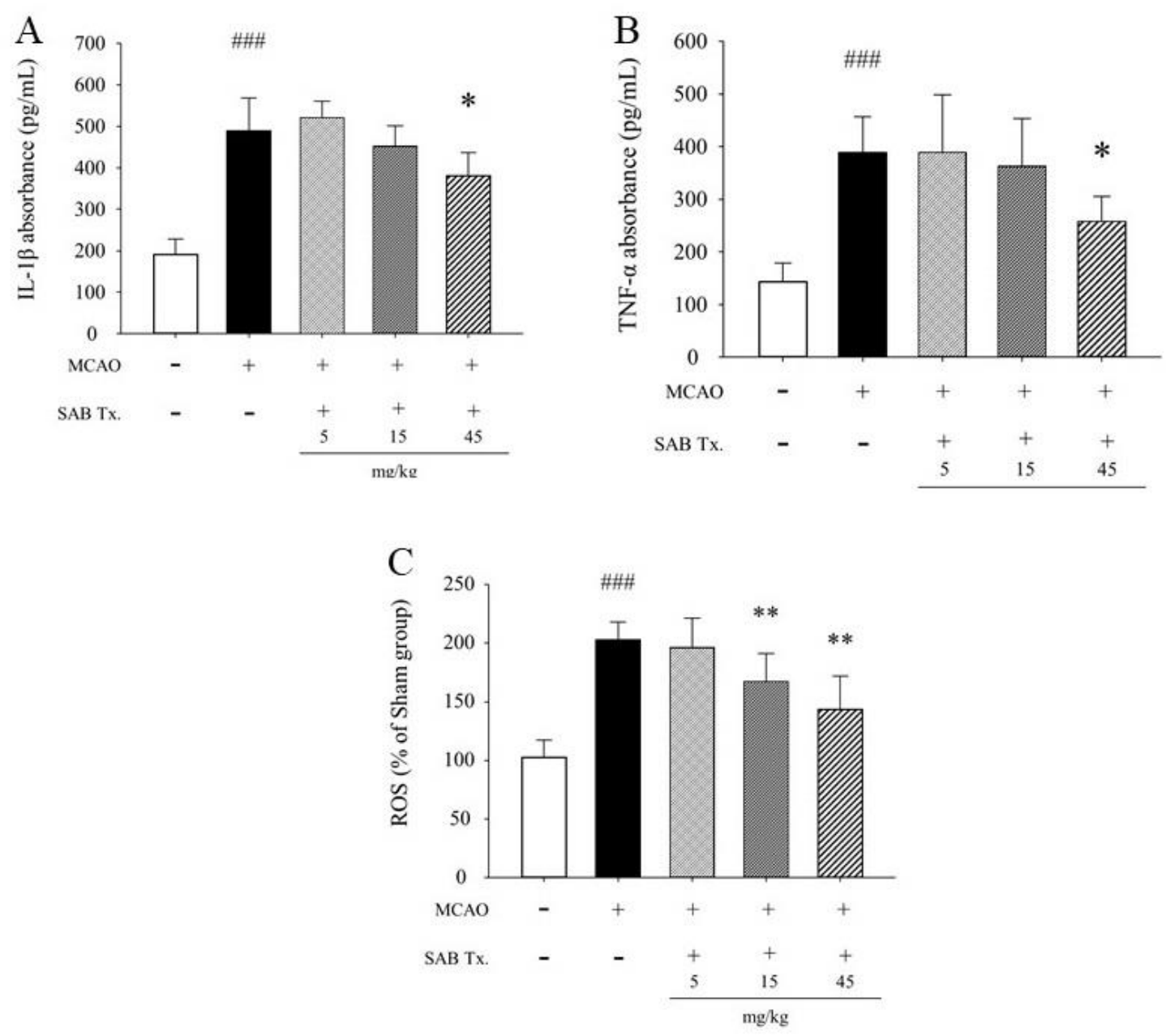

Figure 4. Effects of SAB treatment on IL-1 $\beta$ (A) and TNF- $\alpha(\mathbf{B})$ expressions and ROS (C) production in the ischemic ipsilateral hemispheres of MCAO-induced mice.

$\mathrm{SAB}$ treatment at 3 and $6 \mathrm{~h}$ after MCAO commencement significantly suppressed I/R-induced increases in IL-1 $\beta$, TNF- $\alpha$, and ROS levels. Results are presented as means \pm SDs. \#\#\#p $<0.001$ vs the sham group, ${ }^{*} \mathrm{p}<0.05,{ }^{* *} \mathrm{p}<$ 0.01 vs the MCAO group; $\mathrm{n}=5$ per group.

Thus, inhibitions of IL- $1 \beta$ and TNF- $\alpha$ can protect the brain against ischemic injury, and oral SAB administration suppressed ROS production in brain tissues and inhibited the expressions of proinflammatory cytokines.

\subsection{Effects of SAB on Mn-SOD Protein Expression}

To evaluate the role of Mn-SOD in ischemia-induced brain injury, we measured its protein levels in ischemic ipsilateral hemispheric tissues $24 \mathrm{~h}$ after MCAO operation. MCAO up-regulated Mn-SOD levels, but Mn-SOD protein levels were significantly lower in the SAB 45 group than in the MCAO group (Figure 5).

Mn-SOD is a mitochondrial antioxidant and is activated in mitochondria to detoxify free radicals generated by mitochondrial respiration. Furthermore, the Mn-SOD gene is up-regulated by oxidative stress [22]. We considered the up-regulation of Mn-SOD in ischemic hemispheres was probably due to the generation of ROS, and its down-regulation by SAB was due to the SAB-induced suppression of 
ROS. Overall, our findings indicate SAB suppresses I/R-induced brain damage. We summarize proposed mechanisms responsible for the neuroprotective effects of SAB in Figure 6.

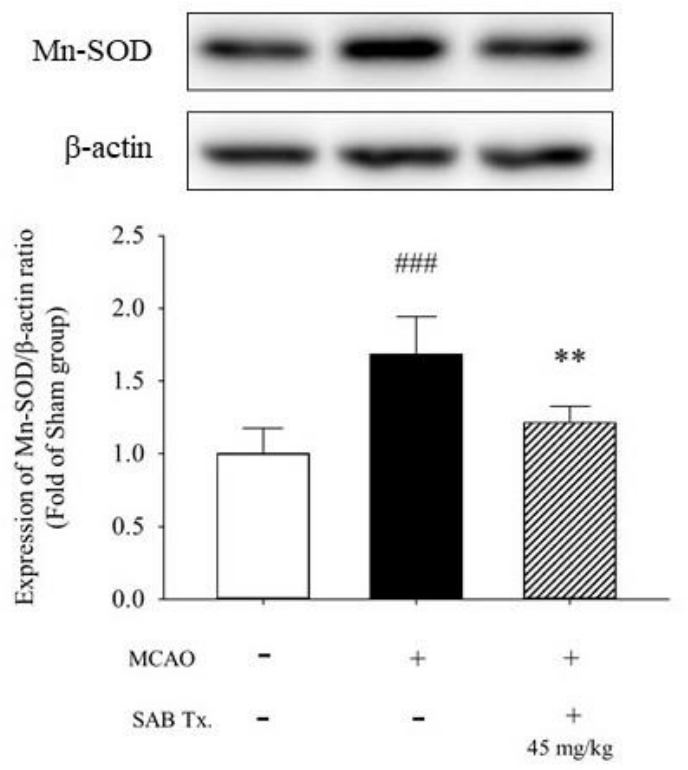

Figure 5. Effects of SAB on Mn-SOD protein levels in ischemic ipsilateral hemispheres of MCAOinduced mice.

SAB treatment after MCAO significantly inhibited I/R-induced increases in Mn-SOD protein levels. Representative western blot analysis result of Mn-SOD protein expression showing the effect of SAB on Mn-SOD expression levels in ipsilateral brain tissues. Results are presented as means \pm SDs. \#\#\#p $<0.001$ vs sham controls, $* * \mathrm{p}<0.01$ vs MCAO controls; $\mathrm{n}=5$ per group.
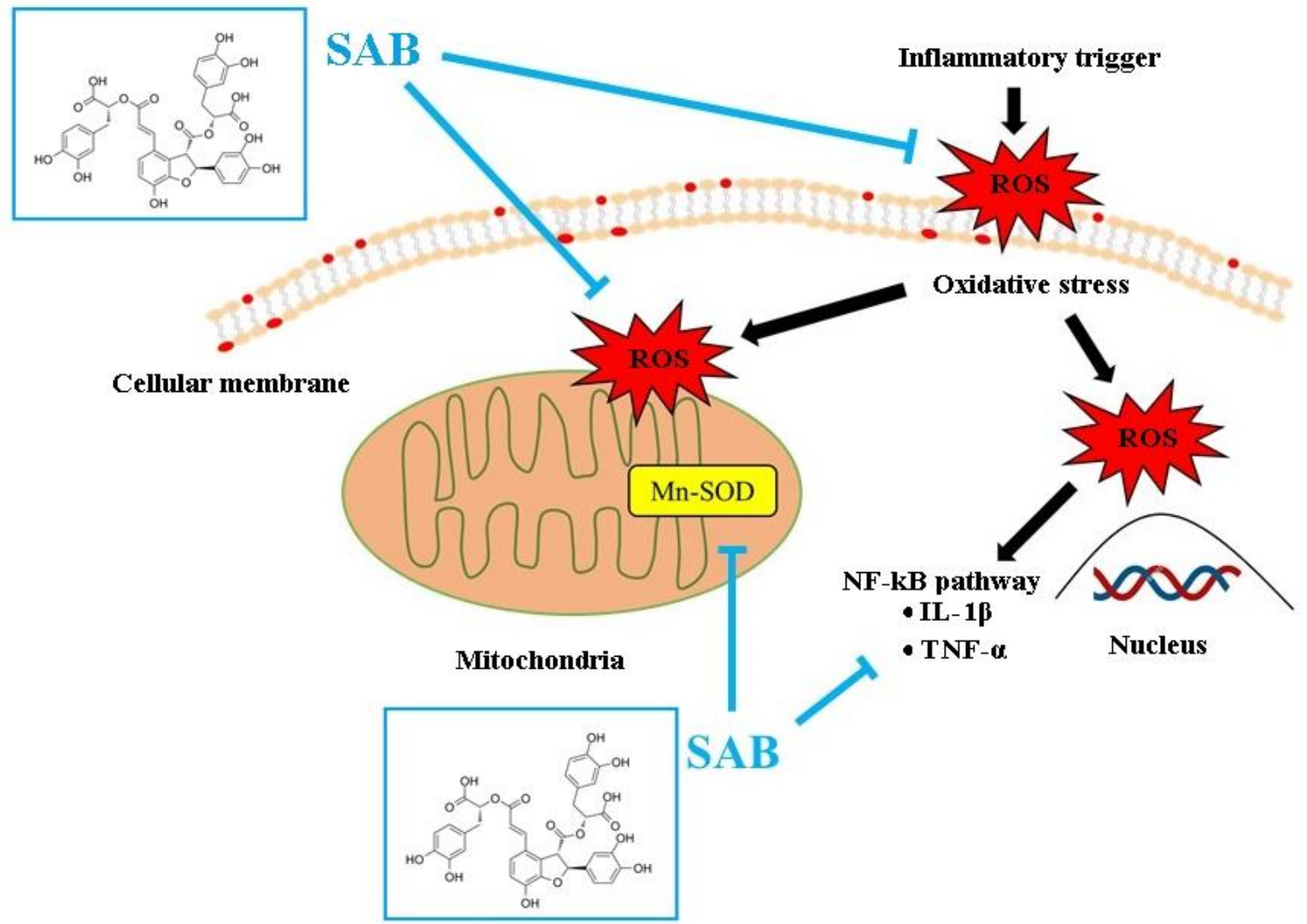

Figure 6. Schematic of our proposed mechanism whereby SAB exhibited a neuroprotective effect in our murine MCAO model. Red bars indicate proposed mechanistic pathways 
As a conclusion, we found that SAB administered after MCAO suppressed ischemia-induced brain injury in our mouse model. SAB-induced reductions in brain infarct volumes were attributed, in part, to reduced brain edema. SAB was found to reduce ROS production and Mn-SOD protein expression in ipsilateral hemispheres, which suggests its potential for the treatment of brain injury. SAB also significantly suppressed the expressions of IL- $1 \beta$ and TNF- $\alpha$ in ischemic brains, suggesting SAB ameliorates I/R-induced brain injury by suppressing inflammatory responses. We believe our results are meaningful because our findings support those of other researchers, who concluded SAB has the potential to suppress brain edema caused by brain injury by attenuating anti-inflammatory responses. Furthermore, the present study provides preclinical evidence regarding the possible therapeutic use of $\mathrm{SAB}$ for the treatment of ischemic stroke.

\section{Supporting Information}

Supporting information accompanies this paper on http://www.acgpubs.org/journal/records-ofnatural-products

\section{ORCID}

Chiyeon Lim: 0000-0003-0178-6976

Chang-Hyun Kim: 0000-0002-8986-1047

Se-Hyun Lim: 0000-0002-2198-661X

Suin Cho: $\underline{0000-0001-8507-1382}$

\section{References}

[1] G. A. Donnan, M. Fisher, M. Macleod and S. M. Davis (2008). Stroke, The Lancet 371, 1612-1623.

[2] V. Hachinski and M. R. Azarpazhooh (2016). Stroke is a burdensome but preventable brain disorder, Lancet Neurol. 15, 892-893.

[3] N. Scherbakov, S. von Haehling, S. D. Anker, U. Dirnagl and W. Doehner (2013). Stroke induced Sarcopenia: muscle wasting and disability after stroke, Int. J. Cardiol. 170, 89-94.

[4] U. Dirnagl (2012). Pathobiology of injury after stroke: the neurovascular unit and beyond, Ann. N. Y. Acad. Sci. 1268, 21-25.

[5] C. Guo, Y. Ma, S. Ma, F. Mu, J. Deng, J. Duan, L. Xiong, Y. Yin, Y. Wang, M. Xi and A. Wen (2017). The role of TRPC6 in the neuroprotection of calycosin against cerebral ischemic injury, Sci. Rep. 7, 3039-01703404-6.

[6] S. Prabhakaran, I. Ruff and R. A. Bernstein (2015). Acute stroke intervention: a systematic review, JAMA 313, 1451-1462.

[7] A. Chamorro, U. Dirnagl, X. Urra and A. M. Planas (2016). Neuroprotection in acute stroke: targeting excitotoxicity, oxidative and nitrosative stress, and inflammation, Lancet Neurol. 15, 869-881.

[8] İ. Gulcin (2020). Antioxidants and antioxidant methods: an updated overview, Arch. Toxicol. 94, 651-715.

[9] K. Malone, S. Amu, A. C. Moore and C. Waeber (2019). Immunomodulatory therapeutic strategies in stroke, Front. Pharmacol. 10, 10.3389/fphar.2019.00630.

[10] F.A.Shah, T. Li, L.T.A. Kury, A. Zeb, S. Khatoon, G. Liu, X. Yang, F. Liu, H. Yao, A.U. Khan, P.O. Koh, Y. Jiang and S. Li (2019). Pathological comparisons of the hippocampal changes in the transient and permanent middle cerebral artery occlusion rat models, Front. Neurol. 10,1178.(14 pages).

[11] Y. Bu, K. Lee, H. S. Jung and S. K. Moon (2013). Therapeutic e.ffects of traditional herbal medicine on cerebral ischemia: a perspective of vascular protection, Chin. J. Integr. Med. 19, 804-814.

[12] F. Mu, J. Duan, H. Bian, Y. Yin, Y. Zhu, G. Wei, Y. Guan, Y. Wang, C. Guo, A. Wen, Y. Yang and M. Xi (2017). Cardioprotective effects and mechanism of Radix Salviae miltiorrhizae and Lignum dalbergiae odoriferae on rat myocardial ischemia/reperfusion injury, Mol. Med. Rep. 16, 1759-1770.

[13] H. Pang, L. Wu, Y. Tang, G. Zhou, C. Qu and J. A. Duan (2016). Chemical analysis of the herbal medicine Salviae miltiorrhizae Radix et rhizoma (Danshen), Molecules 21, 51 (28 pages).

[14] B. Yang, C. Zheng, H. Yu, R. Zhang, C. Zhao and S. Cai (2019). Cardio-protective effects of salvianolic acid B on oxygen and glucose deprivation (OGD)-treated H9c2 cells, Artif. Cells Nanomed. Biotechnol. 47, 2274-2281. 
[15] Y. Fan, Q. Luo, J. Wei, R. Lin, L. Lin, Y. Li, Z. Chen, W. Lin and Q. Chen (2018). Mechanism of salvianolic acid B neuroprotection against ischemia/reperfusion induced cerebral injury, Brain Res. 1679, 125-133.

[16] S. E. Lee, C. Lim, M. Lee, C. H. Kim, H. Kim, B. Lee and S. Cho (2018). Assessing neuroprotective effects of Glycyrrhizae radix et rhizoma extract using a transient middle cerebral artery occlusion mouse model, $J$. Vis. Exp. 142, 10.3791/58454.

[17] Q. Ruan, F. Liu, Z. Gao, D. Kong, X. Hu, D. Shi, Z. Bao and Z. Yu (2013). The anti-inflamm-aging and hepatoprotective effects of huperzine A in D-galactose-treated rats, Mech. Ageing Dev. 134, 89-97.

[18] S. Y. Jung, K. M. Kim, S. Cho, S. Lim, C. Lim and Y. K. Kim (2017). Effects of pretreatment with methanol extract of Peucedani radix on transient ischemic brain injury in mice, Chin. Med. 12, 30-017-0151-z.

[19] E. Naik and V. M. Dixit (2011). Mitochondrial reactive oxygen species drive proinflammatory cytokine production, J. Exp. Med. 208, 417-420.

[20] H. Boutin, R. A. LeFeuvre, R. Horai, M. Asano, Y. Iwakura and N. J. Rothwell (2001). Role of IL-1alpha and IL-1beta in ischemic brain damage, J. Neurosci. 21, 5528-5534.

[21] T. Chiba and K. Umegaki (2013). Pivotal roles of monocytes/macrophages in stroke. Mediators Inflamm. 2013, 759103.

[22] L. Wang, Q. Duan, T. Wang, M. Ahmed, N. Zhang, Y. Li, L. Li and X. Yao (2015). Mitochondrial respiratory chain inhibitors involved in ROS production induced by acute high concentrations of iodide and the effects of SOD as a protective factor, Oxid. Med. Cell. Longev. 2015, 217670.

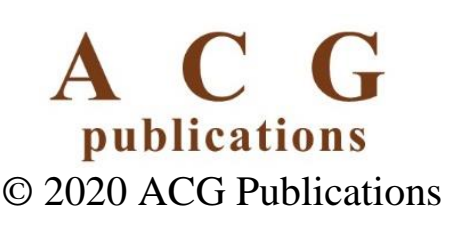

